International Mathematical Forum, Vol. 8, 2013, no. 7, 347 - 355

\title{
Review: Multiple Criteria Decision Making
}

\section{Method with Applications}

\author{
Satinee Lertprapai \\ Department of Mathematics, Faculty of Science \\ Burapha University, Chonburi, Thailand 20131 \\ satineel@buu.ac.th
}

\begin{abstract}
Multiple Criteria Decision Making (MCDM) is a procedure to integrate multiple indicators into a single meaningful index therefore ranking and comparing are feasible. Extending the MCDM method in continuous version is to compare the estimators from distributions in order to find the best estimator.
\end{abstract}

Mathematics Subject Classification: 62F10, 62C25

Keyword: Multiple criteria decision making

\section{Introduction}

The world around us is difficult to see in one-dimensional way in order to judge what we see. We always compare and rank objects of our choice with respect to various criteria of choice, such as countries in regard to their environmental qualities, or several estimators with respect to their mean squared errors. One of the methods to compare, rank and order several alternatives is based on the notion of "Multiple Criteria Decision Making (MCDM)". MCDM has recently been recognized as an efficient statistical method to combine component 'indices' arising from many 'sources' into a single overall meaningful index therefore ranking and comparing are feasible. A typical MCDM problem involves a number of alternatives to be assessed and a number of criteria or indicators to assess the alternatives. Each alternative has a value for each indicator and based on these values the alternatives can be assessed and ranked. Moreover, 
multiple criteria decision making research has developed rapidly and has become a main area of research for dealing with complex decision problems which require the consideration of multiple objectives or criteria.

As advocated by Zeleny [13], and Yoon and Hwang [12], Multiple Criteria Decision Making (MCDM) is a body of techniques used for meaningful integration of component indices to an overall index in order to decide on the ranking of a number of 'locations' from the best to worst. This is based on the premises that in the absence of a natural ideal 'location', a best alternative would be the one which has the shortest distance from the hypothetical ideal 'location' and at the same time farthest distance from the hypothetical anti-ideal (negative ideal) 'location'

In particular, Yoon and Hwang [12] developed an MCDM approach called technique for order preference by similarity to ideal solution (TOPSIS), using the intuitive principle that the best alternatives should have the shortest distance from the ideal alternative and the farthest distance from the negative-ideal alternative. Filar [2] focused on the application of multiple criteria decision making (MCDM) techniques to assess the effectiveness of US environmental policies for the reduction of toxic release. They described in detail the above TOPSIS method. They used entropy as a basis to determine the importance weights and applied the MCDM technique to assess the state and movement of US toxic release of priority chemicals constituting the " $33 / 50$ program".

\section{Description of MCDM procedure}

The basic premise is a data matrix $X=\left(x_{i j}\right): K \times N$ where the rows represent facilities which need to be compared or ranked with respect to the element $x_{i j}$ 's, the columns represent various sources of the elements $x_{i j}$ 's and the $x_{i j}$ 's themselves represent some quantitative information about the facilities. In the context of environmental science, the $x_{i j}$ 's may represent levels of pollutants, facilities represent the sources of the pollutants (e.g., chemical or nuclear facilities) and the columns represent different types of pollution.

The MCDM is a procedure to integrate multiple indicators into a single meaningful and overall index by combining $\left(x_{i 1}, \ldots, x_{i N}\right)$ for row $i$ across all indicators $j=1,2, \ldots, N$. Refer to [12], they define an Ideal Row as one with the smallest observed value for each column

$$
I D R=\left(\min _{i} x_{i 1}, \ldots, \min _{i} x_{i N}\right)=\left(u_{1}, \ldots, u_{N}\right)
$$

and a Negative-ideal Row (NIDR) as one with the largest observed value for each column 


$$
N I D R=\left(\max _{i} x_{i 1}, \ldots, \max _{i} x_{i N}\right)=\left(v_{1}, \ldots, v_{N}\right) .
$$

For any given row $i$, we now compute the distance of each row from Ideal row and from Negative Ideal row based on the $L_{2}$-norm by using the formulae :

$$
\begin{aligned}
& L_{2}(i, I D R)=\left[\sum_{j=1}^{N} \frac{\left(x_{i j}-u_{j}\right)^{2} w_{j}}{\sum_{i=1}^{K} x_{i j}^{2}}\right]^{1 / 2} \\
& L_{2}(i, N I D R)=\left[\sum_{j=1}^{N} \frac{\left(x_{i j}-v_{j}\right)^{2} w_{j}}{\sum_{i=1}^{K} x_{i j}^{2}}\right]^{1 / 2}
\end{aligned}
$$

where $w_{1}, w_{2}, \ldots, w_{N}$ are suitably chosen nonnegative weights between 0 and 1 . An objective way to select the weights [9] is to use Shannon [10]'s entropy measure $\phi$ based on the proportion $p_{1 j}, \ldots, p_{K j}$ for the $j$ th column where

$$
p_{i j}=x_{i j} / \sum_{i=1}^{K} x_{i j} .
$$

For the $j$ th column, $\phi_{j}$ is computed as

$$
\phi_{j}=--\frac{1}{\log K} \sum_{i=1}^{K} p_{i j} \log \left(p_{i j}\right) .
$$

The quantity $\phi$ essentially provides a measure of closeness of the different proportions. The smaller the value of $\phi$, the larger the variation among the proportions for classifying the rows. So we can select the weights as

$$
w_{j}=\left(1-\phi_{j}\right) /\left[\sum_{j=1}^{N}\left(1-\phi_{j}\right)\right], \quad j=1, \ldots, N .
$$

In addition to Shannon's entropy measure, we can also use the sample variance [9] of these proportions, given by

$$
s_{j / \text { prop }}^{2}=\frac{1}{K-1} \sum_{i=1}^{K}\left(p_{i j}-\bar{p}_{j}\right)^{2} .
$$

If $\bar{x}_{j}$ and $s_{j}^{2}$ denote the mean and variance of $x_{i j}$ in the $j$ th column, $s_{j / \text { prop }}^{2}$ is directly proportional to $s_{j}^{2} / \bar{x}_{j}^{2}$, which is the square of the sample coefficient of variation $c v_{j}$ for the $j$ th column. Therefore we propose to use $w_{j}=c v_{j}$ for all $j$. In addition to $L_{2}$-norm we can also use the $L_{1}$-norm as a distance measure and rank the rows once again. The $L_{1}$-norm distance is defined as

$$
L_{1}(i, I D R)=\sum_{j=1}^{N} \frac{\left|x_{i j}-u_{j}\right| w_{j}}{\sum_{i=1}^{K} x_{i j}}
$$




$$
L_{1}(i, N I D R)=\sum_{j=1}^{N} \frac{\left|x_{i j}-v_{j}\right| w_{j}}{\sum_{i=1}^{K} x_{i j}}
$$

where $w_{j}{ }^{\prime} s$ are appropriate weights.

The various rows are now ranked based on an overall index $I$ computed as

$$
I_{i}=\frac{L_{2}(i, I D R)}{L_{2}(i, I D R)+L_{2}(i, N I D R)}, \quad i=1, \ldots, K
$$

for $L_{2}$-norm and

$$
I_{i}=\frac{L_{1}(i, I D R)}{L_{1}(i, I D R)+L_{1}(i, N I D R)}, \quad i=1, \ldots, K
$$

for $L_{1}$-norm.

In 2002, Maitra [9] discussed some extensions and generalizations of MCDM, and applied MCDM to data on air, water and land quality of the fifty US states, and similar indices for 106 countries in the United Nations human environmental indicators (HEI) study. In 2004, Lertprapai [4] applied MCDM method to the air pollution data measured as carbon monoxide, nitrogen dioxide, sulfur dioxide and ozone from ten monitoring stations in Bangkok, Thailand to compute an overall air pollution index for these stations and compare them. Moreover, there are many other applications of MCDM in data quality see $[7,8]$.

\section{Comparison of the estimators}

There are many researches involved the problem of comparing and ranking estimators using various methods. But Lertprapai [5] are interested in the problem of comparing and ranking estimators based on MCDM method. They set up a 'continuous' version involve $x_{i j}$ 's where the index $j$ would vary 'continuously'. In the context of an estimation problem, the $x_{i j}$ 's may represent mean squared errors of different estimators, designated by rows, for different values of the unknown parameter, denoted by $\theta$, designated by columns. So the $L_{1}$-norm and $L_{2}$-norm would be redefined as

$$
\begin{aligned}
L_{1}(i, I D R) & =\int_{\underline{\theta}}^{\bar{\theta}}\left[x_{i}(\theta)-u(\theta)\right] w(\theta) d \theta \\
L_{1}(i, N I D R) & =\int_{\underline{\theta}}^{\bar{\theta}}\left[v(\theta)-x_{i}(\theta)\right] w(\theta) d \theta
\end{aligned}
$$




$$
\begin{aligned}
& L_{2}(i, I D R)=\sqrt{\sqrt{\int_{\underline{\theta}}^{\theta}}\left(x_{i}(\theta)-u(\theta)\right)^{2} w(\theta) d \theta} \\
& L_{2}(i, N I D R)=\sqrt{\int_{\underline{\theta}}^{\theta}\left(x_{i}(\theta)-v(\theta)\right)^{2} w(\theta) d \theta}
\end{aligned}
$$

where $w(\theta)$ is weight function, $u(\theta)$ is ideal row defined as $u(\theta)=\min _{i}\left\{x_{i}(\theta)\right\}$ and $v(\theta)$ is negative ideal row defined as $v(\theta)=\max _{i}\left\{x_{i}(\theta)\right\}$, when $\underline{\theta} \leq \theta \leq \bar{\theta}$.

The problem of comparing and ranking estimators on the basis of MCDM as the following.

In 2004 Lertprapai [5] focused on two standard estimates of $\theta$ based on binomial distribution, recall that $X \sim B(n, \theta)$. There are two estimators for mean $\theta$, that is $T_{1}(X)=X / n$, the maximum likelihood estimate and $T_{2}(X)=[X+\sqrt{n} / 2] /[n+\sqrt{n}]$, the minimax estimate under the squared error loss function [1], are some estimates of $\theta$ which are compared with respect to their mean squared errors (MSE) where $\operatorname{MSE}\left(T_{1}\right)=\frac{\theta(1-\theta)}{n}$ and $\operatorname{MSE}\left(T_{2}\right)=$ $\frac{n}{4(n+\sqrt{n})^{2}}$. Let $\operatorname{MSE}\left(T_{i}\right)=x_{i}(\theta), i=1, \ldots, K$, where $\underline{\theta} \leq \theta \leq \bar{\theta}$. They consider two additional choices of $w(\theta)$. The first one, denoted by $w_{1}(\theta)$, is based on the notion of entropy between $\operatorname{MSE}\left(T_{1}\right)$ and $\operatorname{MSE}\left(T_{2}\right)$ for various values of $\theta$, and the second one, denoted by $w_{2}(\theta)$, is based on the coefficient of variation of MSE $\left(T_{1}\right)$ and $\operatorname{MSE}\left(T_{2}\right)$ for various values of $\theta$. It then readily turns out that

$$
w_{1}(\theta)=\frac{1-\varphi(\theta)}{\int_{\underline{\theta}}^{\bar{\theta}}[1-\varphi(\theta)] d \theta}
$$

where

$$
\phi(\theta)=-\frac{1}{\log 2}\left\{\left(\frac{\frac{\theta(1-\theta)}{n}}{\frac{\theta(1-\theta)}{n}+\frac{n}{4(n+\sqrt{n})^{2}}}\right) \cdot \log \left(\frac{\frac{\theta(1-\theta)}{n}}{\frac{\theta(1-\theta)}{n}+\frac{n}{4(n+\sqrt{n})^{2}}}\right)+\right.
$$




$$
\left.\left(\frac{\frac{n}{4(n+\sqrt{n})^{2}}}{\frac{\theta(1-\theta)}{n}+\frac{n}{4(n+\sqrt{n})^{2}}}\right) \cdot \log \left(\frac{\frac{n}{4(n+\sqrt{n})^{2}}}{\frac{\theta(1-\theta)}{n}+\frac{n}{4(n+\sqrt{n})^{2}}}\right)\right\}
$$

and

$$
w_{2}(\theta)=\frac{\left|\frac{\theta(1-\theta)}{n}-\frac{n}{4(n+\sqrt{n})^{2}}\right|}{\frac{\theta(1-\theta)}{n}+\frac{n}{4(n+\sqrt{n})^{2}}} .
$$

The results are stated below.

Corollary 3.1 : Let $\theta$ be a binomial proportion, $0<\theta<1$. Under $w_{1}(\theta), T_{2}(X)$ is better than $T_{1}(X)$ for all $n \geq 1$.

Corollary 3.2 : Let $\theta$ be a binomial proportion, $0<\theta<1$. Under $w_{2}(\theta), T_{2}(X)$ is better than $T_{1}(X)$ for all $n \in[1,19]$.

In the case of $L_{2}$-norm with a general weight function $w(\theta)$, proceeding as before,

Theorem 3.1 : Let $\theta$ be a binomial proportion, $0<\theta<1$. If the weight function is defined by $w(\theta)=\theta^{\alpha-1}(1-\theta)^{\beta-1}$ for some $\alpha, \beta>0$, then $T_{2}(X)$ is better than $T_{1}(X)$ based on $L_{2}$-norm.

Corollary 3.3 : Let $\theta$ be a binomial proportion, $0<\theta<1$. If the weight function is defined by (17) then $T_{2}$ is better than $T_{1}$ based on $L_{2}$-norm for all $n \geq 1$.

Corollary 3.4 : Let $\theta$ be a binomial proportion, $0<\theta<1$. If the weight function is defined by (18) then $T_{2}$ is better than $T_{1}$ based on $L_{2}$-norm if $n \in[1,14]$.

Based on the above analysis under $L_{1}$ - and $L_{2}$ - norms, their recommendation is to use $T_{2}$ rather than $T_{1}$ for small values of $n$.

In addition to binomial distribution, $B(n, \theta)$, Lertprapai [6] compared three estimators of $\theta(1-\theta)$, namely, $T_{1}=\frac{X}{n}\left(1-\frac{X}{n}\right)$, the maximum likelihood estimate [8], $T_{2}=\frac{X(n-X)}{n(n-1)}$, the uniformly minimum variance unbiased estimate [1] and $T_{3}=\frac{X(n-X)+n \sqrt{n} / 2+n / 4}{(n+\sqrt{n})^{2}}$, based on the minimax estimate of $\theta$. $x_{i}(\theta), i=1,2,3$ are chosen to represent the mean squared errors of $T_{1}, T_{2}$ and $T_{3}$ 
respectively for various values of $\theta$, and $L_{1}$-norm and $L_{2}$-norm would be redefined as equation (13)-(16). First weight function $w_{1}(\theta)$ is defined by $w_{1}(\theta)=\frac{\theta^{\alpha-1}(1-\theta)^{\beta-1}}{B(\alpha, \beta)}$ for some $\alpha, \beta>0$, which is a conjugate prior for the binomial parameter $\theta$. They also consider two additional choices of $w(\theta)$. The first one, denoted by $w_{2}(\theta)$, is based on the notion of entropy among $x_{1}(\theta)$, $x_{2}(\theta)$ and $x_{3}(\theta)$ for various values of $\theta$, and the second one, denoted by $w_{3}(\theta)$, is based on the coefficient of variation of $x_{1}(\theta), x_{2}(\theta)$ and $x_{3}(\theta)$ for various values of $\theta$. After apply MCDM method using $L_{1}$-norm and $L_{2}$-norm as well as three choices of weight functions. The results show that for small values of $n$, their preference is uniform for $T_{1}$. Although $T_{2}$ is uniformly the minimum variance unbiased estimator (UMVUE), it should be the best one. However $T_{2}$ is improper since $T_{2}(x)>1 / 4$ whenever $(n-\sqrt{n}) / 2<x<(n+\sqrt{n}) / 2$. Therefore they use a truncated version $T_{2}^{*}$ of $T_{2}$ and compare it with the other two. The result shows that the performance of $T_{2}^{*}$ is better.

In 2005 Lertprapai [11] considered the problem of estimation of the mean $\mu$ from a normal population $N\left(\mu, \sigma^{2}\right)$ and assume that the uncertain non-sample prior information on the value of $\mu$ is available. They refer to Khan and Saleh [3] to get three biased estimators of $\mu$, namely, $T_{2}: \hat{\mu}_{n}(d)=d \tilde{\mu}_{n}+(1-d) \mu_{0}, \quad 0 \leq d \leq 1$ , the restricted estimator (RE), $T_{3}: \hat{\mu}_{n}^{P T E}(d)=\hat{\mu}_{n}(d) I\left(\left|t_{v}\right|<t_{\alpha / 2}\right)+\tilde{\mu}_{n} I\left(\left|t_{v}\right| \geq t_{\alpha / 2}\right)$, the preliminary test estimator (PTE) and $T_{4}: \hat{\mu}_{n}^{s}=\mu_{0}+\left\{1-\frac{c S_{n}}{\left|\sqrt{n}\left(\tilde{\mu}_{n}-\mu_{0}\right)\right|}\right\}\left(\tilde{\mu}_{n}-\mu_{0}\right)$, the shrinkage estimator (SE). Besides these three estimators, they also consider the unbiased estimator $T_{1}: \tilde{\mu}_{n}=\bar{x}$. They compare the unbiased estimator $\bar{x}$ and the three biased estimators based on mean square errors (MSE) by using Multiple Criteria Decision Making (MCDM) method for searching the best estimator. First weight function $w_{1}(\theta)$ is defined by $w_{1}(\theta)=1$. They also consider two additional choices of $w(\theta)$. The first one, denoted by $w_{2}(\theta)$, is based on the notion of entropy among $x_{1}(\theta), x_{2}(\theta), x_{3}(\theta)$ and $x_{4}(\theta)$ for various values of $\theta$, and the second one, denoted by $w_{3}(\theta)$, is based on the coefficient of variation of $x_{1}(\theta), x_{2}(\theta), x_{3}(\theta)$ and $x_{4}(\theta)$ for various values of $\theta$. They report the ranks of the four estimates when compared using $L_{1}$-norm and $L_{2}$-norm on the basis of the weight functions $w_{1}(\theta), w_{2}(\theta)$ and $w_{3}(\theta)$. It turns out that among the biased estimators, the superior estimator belongs to $T_{4}$ and most of the last rank belongs to $T_{3}$. 


\section{Conclusion}

This article presents a methodology for decision making based on Multiple Criteria Decision Making (MCDM) method. MCDM is a procedure to integrate multiple indicators or various columns of a data matrix into a single meaningful result, thus making a ranking of the rows and hence their comparison feasible at the end of this part. Extending the Multiple Criteria Decision Making (MCDM) method in continuous version is to compare the estimators from distributions in order to obtain the best one.

\section{References}

[1] G. Casella, and R.L. Berger, Statistical Inference, Wadsworth Inc, California, 1990.

[2] J.A. Filar, N.P. Ross, and M.L. Wu, Environmental Assessment Based on Multiple Indicators. Technical Report, Department of Applied Mathematics, University of South Australia (1999), 141-149.

[3] S. Khan, and A.K. Saleh, On the comparison of the pre-test and shrinkage estimators for the univariate normal mean, Statistical Papers 42 (2001), 541473.

[4] S. Lertprapai, M. Tiensuwan, and B.K. Sinha, A statistical approach to combining environmental indices with and application to air pollution data from Bangkok, Thailand, Pakistan Journal of Statistics 20/2 (2004), 245-261.

[5] S. Lertprapai, M. Tiensuwan, and B.K. Sinha, On a comparison of two standard estimates of binomial proportion based on Multiple Criteria decision making method, Journal of Statistical Theory and Applications 3 (2004), 141-149.

[6] S. Lertprapai, M. Tiensuwan, and B.K. Sinha, On a comparison of three estimators of binomial variance by multiple criteria decision making method, International Journal of Statistical Sciences 3 (2004), 105-117.

[7] S. Lertprapai and M. Tiensuwan, An application of multiple criteria decision making in combining environmental indices of five air pollution indicators, Thailand Statistician 7/2 (2009), 131-145. 
[8] S. Lertprapai and M. Tiensuwan, An application of multiple criteria decision making to water quality data in Thailand, Far East Journal of Applied Mathematics 40/2 (2010), 103-124.

[9] R. Maitra, N.P. Ross, and B.K. Sinha, On some aspects of Data integration Techniques with Application, Technical report. Department of Mathematics and Statistics, UMBC, USA, 2002.

[10] C.E. Shannon, W. Weaver, The Mathematical theory of communication, The University of Illinois Press, Urbana, Illinois, 1947

[11] M. Tiensuwan, S. Lertprapai, S and B.K. Sinha, On a comparison of several competing estimates of a univariate normal mean by the Multiple Criteria Decision Making method, Communications in Statistics-Simulation and Computation 35 (2006), 877-891.

[12] K. Yoon, and C.L. Hwang, Multiple Attribute Decision Making, An Introduction. Sage, California.

[13] M. Zeleny, Multiple criteria decision making, McGraw-Hill, New York, 1982. 